Análisis de sensibilidad de las condiciones operacionales, geológicas y el cuidado post inyección en sitio con el riesgo asociado al secuestro de CO2 en la región sur de Estados Unidos

Danilo Arcentales-Bastidas, Kenny Escobar-Segovia, Bryan Medina-Rodríguez, Romel Erazo-Bone, Carlos Portilla, Carlos Malavé, Alamir Álvarez, Dario Cedeño. 


\title{
Análisis de sensibilidad de las condiciones operacionales, geológicas y el cuidado post inyección en sitio con el riesgo asociado al secuestro de $\mathrm{CO}_{2}$ en la región sur de Estados Unidos
}

\author{
Sensitivity analysis of the impacts of operational and geologic conditions \\ on Area of Review (AOR), Post Injection Site Care (PISC) and Risk \\ associated with $\mathrm{CO}_{2}$ Sequestration in South-region of United States
}

(a) Danilo Arcentales-Bastidas, (a) Kenny Escobar-Segovia, (a) Bryan Medina-Rodríguez, (a) Romel Erazo-Bone,

(b) Carlos Portilla Lazo, (b) Carlos Malavé Carrera, (b) Alamir Álvarez Loor, (b) Dario Cedeño Macias.

(a) Escuela Superior Politécnica del Litoral, Facultad de Ingeniería en Ciencias de la Tierra, Campus "Gustavo Galindo V.”, Km. 30.5, vía Perimetral Apartado 09-01-5863, Guayaquil, Ecuador

(b) Universidad Estatal Península de Santa Elena (UPSE)

\begin{abstract}
Resumen
Para la captura de dióxido de carbono antropogénico $\left(\mathrm{CO}_{2}\right)$ se debe considerar: la capacidad de almacenamiento de gas de la formación, el tamaño de la pluma de saturación y presión durante el monitoreo de la locación luego de la inyección; incluyendo los riesgos asociados con las fugas de $\mathrm{CO}_{2}$ y la reactivación de fallas. Una formación con un volumen poroso razonable sería un buen candidato para el almacenamiento de $\mathrm{CO}_{2}$, sin embargo, no todas las formaciones de alta porosidad tienen la capacidad de almacenar grandes cantidades de gas durante un largo periodo de tiempo. Esa es la mayor preocupación cuando se habla de la captura de $\mathrm{CO}_{2}$. El tamaño de la pluma de saturación y de presión durante la inyección de $\mathrm{CO}_{2}$, así como el control de la locación luego de la inyección, fueron simulados en esta investigación, a través del uso de modelos de yacimientos del campo CRD. La aplicación de diagramas de Pareto y respuestas de superficie permitieron determinar los parámetros más importantes que afectaron a la pluma de saturación y de presión, cuantificando la correlación entre diferentes parámetros de modelos históricos ajustados y dimensionados.
\end{abstract}

Palabras Clave: Captura de $\mathrm{CO}_{2}$, diseño experimental Plackett-Burman, Método de muestreo, Latin Hypercube, gráficos de Pareto.

\begin{abstract}
For anthropogenic carbon dioxide $\left(\mathrm{CO}_{2}\right)$ capture is important to consider: gas storage's formation capacity, saturation and pressure plume size after injection; including the risks associated with $\mathrm{CO}_{2}$ leakage and faults reactivation. A formation with a reasonable pore volume would be a good candidate for $\mathrm{CO}_{2}$ storage, however, not all high porosity formations have the ability to store large amounts of gas over a long period of time. That's the biggest concern when it refers to $\mathrm{CO}_{2}$ capture. Saturation and pressure plume size during $\mathrm{CO}_{2}$ injection as well as site monitoring after injection were simulated in this research, using CRD field reservoir models. The application of Pareto diagrams and surface responses allowed us to determine the most important parameters that affected the saturation and pressure plume, quantifying the correlation between different parameters of adjusted and dimensioned historical models.
\end{abstract}

Keywords: $\mathrm{CO}_{2}$ sequestration, Plackett-Burman experimental design, Latin Hypercube sampling method, Pareto charts

\section{Introducción}

El rápido crecimiento de la población y de la economía ha incrementado la demanda de energía y como consecuencia la demanda en combustibles, especialmente en combustibles fósiles. Esto ha provocado efectos negativos al medio ambiente, relacionados a las emisiones de dióxido de carbono $\mathrm{CO}_{2}$. Según Emission Database for Global Atmospheric Research, las emisiones de $\mathrm{CO}_{2}$ incrementaron en un $39 \%$ en el último siglo. ${ }^{[1]}$
Durante los últimos años, se han encontrado algunos prospectos adecuados para el almacenamiento de $\mathrm{CO}_{2}$ a largo plazo, tales como yacimientos de petróleo y gas depletados, formaciones salinas a grandes profundidades y yacimientos off-shore. ${ }^{[2] ~[3] ~}$

La captura de $\mathrm{CO}_{2}$ en este análisis implica la inyección de gases de efecto invernadero en fase supercrítica, en formaciones geológicas que deben ser estudiadas exhaustivamente. En el desarrollo de este trabajo se seleccionó un acuífero salino de gran 
profundidad con el fin de capturar y almacenar el $\mathrm{CO}_{2}$ de la forma adecuada. Este acuífero salino fue delimitado por una roca impermeable en la parte superior con el fin de evitar emisiones de flujo hacia la superficie que puedan provocar daños al ambiente. Debido a su alta salinidad y profundidad, la explotación del agua contenida en estos acuíferos no es económicamente viable. ${ }^{[4]}$

Existe una inminente necesidad de desarrollar un modelo que describa cómo varía el comportamiento de las métricas de riesgo en función del tamaño, ambiente geológico, volumen y tiempo de inyección. Para este propósito, el método de muestreo Latin Hypercube Sampling (LHS) se utilizó para obtener una amplia gama de diferentes condiciones y generar una matriz de simulación que varíe en función de múltiples parámetros. [5]

El objetivo de esta investigación es desarrollar un procedimiento que ejemplifique estudios de captura de $\mathrm{CO}_{2}$ donde se considere la influencia de todos los escenarios y condiciones relevantes abordando

\begin{tabular}{|c|c|c|c|c|c|}
\hline $\begin{array}{l}\frac{n}{n} \\
\frac{1}{0} \\
\frac{1}{0}\end{array}$ & $\frac{\mathscr{D}}{\stackrel{\mathscr{D}}{\bar{D}}}$ & Unidad Estratigráfica & \multicolumn{2}{|c|}{ Sub-unidad Mayor } & $\begin{array}{c}\text { Potenciales } \\
\text { reservorios y zonas de } \\
\text { confinamiento }\end{array}$ \\
\hline \multirow{8}{*}{$\frac{0}{\text { का. }}$} & कृ & & \multicolumn{2}{|c|}{ Formación CF } & Acuífero de agua dulce \\
\hline & 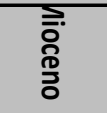 & Indiferenciado & & & Acuífero de agua dulce \\
\hline & 品 & & \multicolumn{2}{|c|}{ Formación CWY } & Base de USDW \\
\hline & 只 & Grupo V & \multicolumn{2}{|c|}{ Arcilla BCT } & $\begin{array}{l}\text { Unidad local de } \\
\text { confinamiento }\end{array}$ \\
\hline & \multirow{2}{*}{ 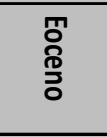 } & Grupo J & & & $\begin{array}{c}\text { Reservorio de menor } \\
\text { salinidad }\end{array}$ \\
\hline & & Grupo C & \multicolumn{2}{|c|}{ Formación TLH } & Reservorio salino \\
\hline & \multirow{2}{*}{$\begin{array}{l}\text { D } \\
\frac{0}{D} \\
0 \\
\frac{0}{0} \\
0\end{array}$} & Grupo W & \multicolumn{2}{|c|}{$\begin{array}{c}\text { Arena HTC BM Domo salino } \\
\text { LS }\end{array}$} & Reservorio salino \\
\hline & & Grupo M & \multicolumn{2}{|c|}{ Arcilla РTCK } & $\begin{array}{c}\text { Unidad de } \\
\text { confinamiento }\end{array}$ \\
\hline \multirow{5}{*}{ 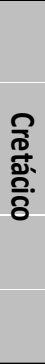 } & \multirow{5}{*}{$\begin{array}{l}\text { 등 } \\
\text { 욤. } \\
\text { 음 }\end{array}$} & Grupo S & & & $\begin{array}{c}\text { Unidad de } \\
\text { confinamiento }\end{array}$ \\
\hline & & Formación E & & & $\begin{array}{c}\text { Reservorio de menor } \\
\text { salinidad }\end{array}$ \\
\hline & & \multirow{3}{*}{ Grupo T } & T Superior & & $\begin{array}{c}\text { Reservorio de menor } \\
\text { salinidad }\end{array}$ \\
\hline & & & T Intermedia & Lutita marina & $\begin{array}{c}\text { Unidad de } \\
\text { confinamiento }\end{array}$ \\
\hline & & & T Inferior & $\begin{array}{l}\text { Arena Piloto } \\
\text { Arena Masiva }\end{array}$ & Reservorio salino \\
\hline \multirow{8}{*}{ 高 } & \multirow{8}{*}{$\begin{array}{l}\overline{\bar{D}} \\
\text { 离. } \\
\text { 윽 }\end{array}$} & \multirow[b]{2}{*}{$\mathbf{W}-\mathbf{F}$} & \multirow{2}{*}{\multicolumn{2}{|c|}{ Arena D y lutita Basal }} & Reservorio salino \\
\hline & & & & & $\begin{array}{c}\text { Unidad de } \\
\text { confinamiento }\end{array}$ \\
\hline & & Formación P & \multicolumn{2}{|c|}{$\begin{array}{c}\text { Superior' } \\
\text { 'Intermedia' } \\
\text { 'Inferior' } \\
\end{array}$} & $\begin{array}{l}\text { Zona propuesta de } \\
\text { inyección }\end{array}$ \\
\hline & & Formación MS & & & $\begin{array}{c}\text { Unidad de } \\
\text { confinamiento }\end{array}$ \\
\hline & & Anhidrita FL & & & $\begin{array}{c}\text { Unidad de } \\
\text { confinamiento }\end{array}$ \\
\hline & & \multirow{3}{*}{ Arena DN } & \multirow{3}{*}{\multicolumn{2}{|c|}{$\begin{array}{l}\text { Superior' } \\
\text { 'Intermedia' } \\
\text { 'Inferior' }\end{array}$}} & $\begin{array}{c}\text { Reservorio de } \\
\text { petróleo }\end{array}$ \\
\hline & & & & & $\begin{array}{c}\text { Reservorio de menor } \\
\text { salinidad }\end{array}$ \\
\hline & & & & & $\begin{array}{c}\text { Reservorio de } \\
\text { petróleo }\end{array}$ \\
\hline
\end{tabular}

Figura 1. Columna estratigráfica del campo CRD. Fuente: Prueba antropogénica SECARB: Prueba integrada de captura, transporte y almacenamiento de $\mathrm{CO}_{2}$ en EE.UU. 
cuestiones como el tamaño de la pluma de presión y saturación, y el monitoreo en la locación después de la inyección. Dos modelos diferentes fueron utilizados para este análisis, el primero con ajuste histórico de producción $(5 \mathrm{~km} \quad \mathrm{x} \quad 5 \mathrm{~km})$ y el segundo sobredimensionado $(10 \mathrm{~km} \times 10 \mathrm{~km})$ manteniendo la misma estructura de formación del modelo ajustado.

\section{Descripción del campo}

El presente estudio se llevó a cabo en el Campo CRD considerando un domo salino libre de fallas, que forma un cierre estructural elíptico, mostrando adecuadas características para la captura de $\mathrm{CO}_{2}$.

El pozo analizado es el D-9-7, ubicado en la Unidad Sureste del campo, a través del cual se inyectó $\mathrm{CO}_{2}$ proveniente de una planta de generación eléctrica a base de carbón; El $\mathrm{CO}_{2}$ fue transportado al lugar de almacenamiento por un ducto de doce millas de longitud.

\subsection{Descripción geológica}

La formación $\mathrm{P}$, situada a una profundidad aproximada de 9.400 a 10.500 pies (TVD), consta de 1.100 pies de arenisca intercalada con limolita y lutita. Esta formación está separada por dos extensas capas de lutita provenientes de la arena W-F (reservorio salino) en la parte superior y la arena DN (reservorio de petróleo) en la parte inferior. De acuerdo a la bibliografía del campo se conoce que existen 17 capas de arenisca que fueron correlacionadas mediante registros petrofísicos y datos de núcleos ${ }^{[6]}$. Además, por encima de la formación W$\mathrm{F}$ se encuentra el grupo $\mathrm{T}$, cuyo acuífero salino profundo contiene múltiples unidades geológicas de confinamiento que sirven de barrera para evitar fugas de $\mathrm{CO}_{2}$ a superficie, siendo una de las varias formaciones de arenisca bien selladas en esta locación.
Estudios anteriores acerca de este método de almacenamiento destacan que, los reservorios salinos de edad Cretácico Superior pueden almacenar una capacidad de $\mathrm{CO}_{2}$ por casi un siglo. Las arenas Masiva y Piloto, del Grupo T inferior, así como varias unidades de arenisca en el Grupo $\mathrm{T}$ superior y formación E, contienen potenciales sumideros de carbono (Figura 1). Estas unidades de arenisca poseen la notable característica de tener porosidad y permeabilidad alta, y una baja heterogeneidad. ${ }^{[7][8]}$

En la formación $\mathrm{P}$ superior, las areniscas que poseen superficies de fondo irregulares, son características de depósitos de arena de origen fluvial que resultan de procesos de agradación sedimentaria.

\section{Metodología}

Con el fin de desarrollar un modelo heterogéneo que se asemeje a la geología del yacimiento, se generaron mapas de porosidad mediante la interpretación de 40 registros de pozos existentes que rodean el área de la locación del pozo. Después de reunir toda esta información, estos 40 puntos de control fueron graficados con métodos geoestadísticos, mediante la aplicación de la ecuación de Archie.

$$
\phi=\left(\frac{a}{\left(\frac{h^{\prime} t}{k^{\prime} w}\right)+S w^{i n}}\right)^{1 / m}
$$

\section{Ec.1}

La ecuación 1 se utiliza con el fin de calcular los valores de porosidad, utilizando el espesor de cada capa considerada Basado en el gradiente térmico de la región, la temperatura de depósito de esta formación se estableció como $110^{\circ} \mathrm{C}$ aproximadamente. Como propósito de explicar de mejor manera la metodología empleada en este estudio, se presenta la figura 2:
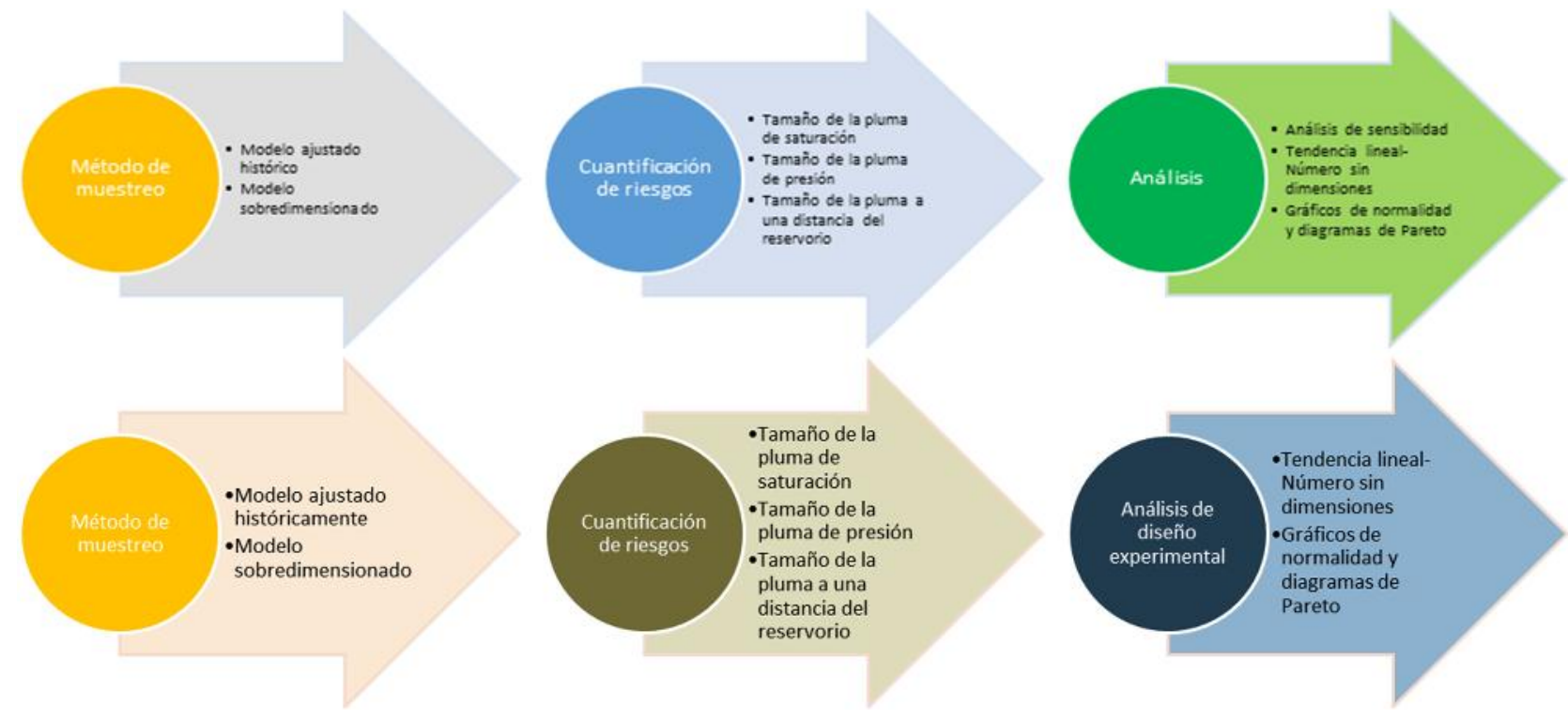

Figura 2. Secuencia metodológica. 


\subsection{Cuantificación de métricas de riesgos}

Se realizó un análisis de la expansión de presión y pluma de $\mathrm{CO}_{2}$ durante y después de la inyección. Por esta razón, tres métricas de riesgo fueron identificadas para evaluar y analizar el comportamiento del reservorio: el tamaño de la pluma de saturación, la pluma de presión diferencial y la presión diferencial en lugares específicos del reservorio como se observa en la Figura 3.

Cabe indicar que se asignaron diferentes umbrales de presión y de saturación con el objetivo de estudiar el tamaño de la pluma de $\mathrm{CO}_{2}$ y el área de presión. Estos umbrales son: 1,5 y 10 bar para la pluma de presión y 0,01 y 0,2 para la pluma de saturación de $\mathrm{CO}_{2}$.

3.1.1. Pluma de saturación de $\mathrm{CO}_{2}$ : La pluma de saturación de $\mathrm{CO}_{2}$ en la Figura 4 nos muestra la evolución de su tamaño durante y después del periodo de inyección. Como se observa en la figura, el tamaño de la pluma se expandió rápidamente durante el período de inyección y luego de la inyección se ralentiza. La tasa de crecimiento de la pluma a ambos tiempos, temprano y tardío, se caracterizaron con las pendientes m1 y m2:

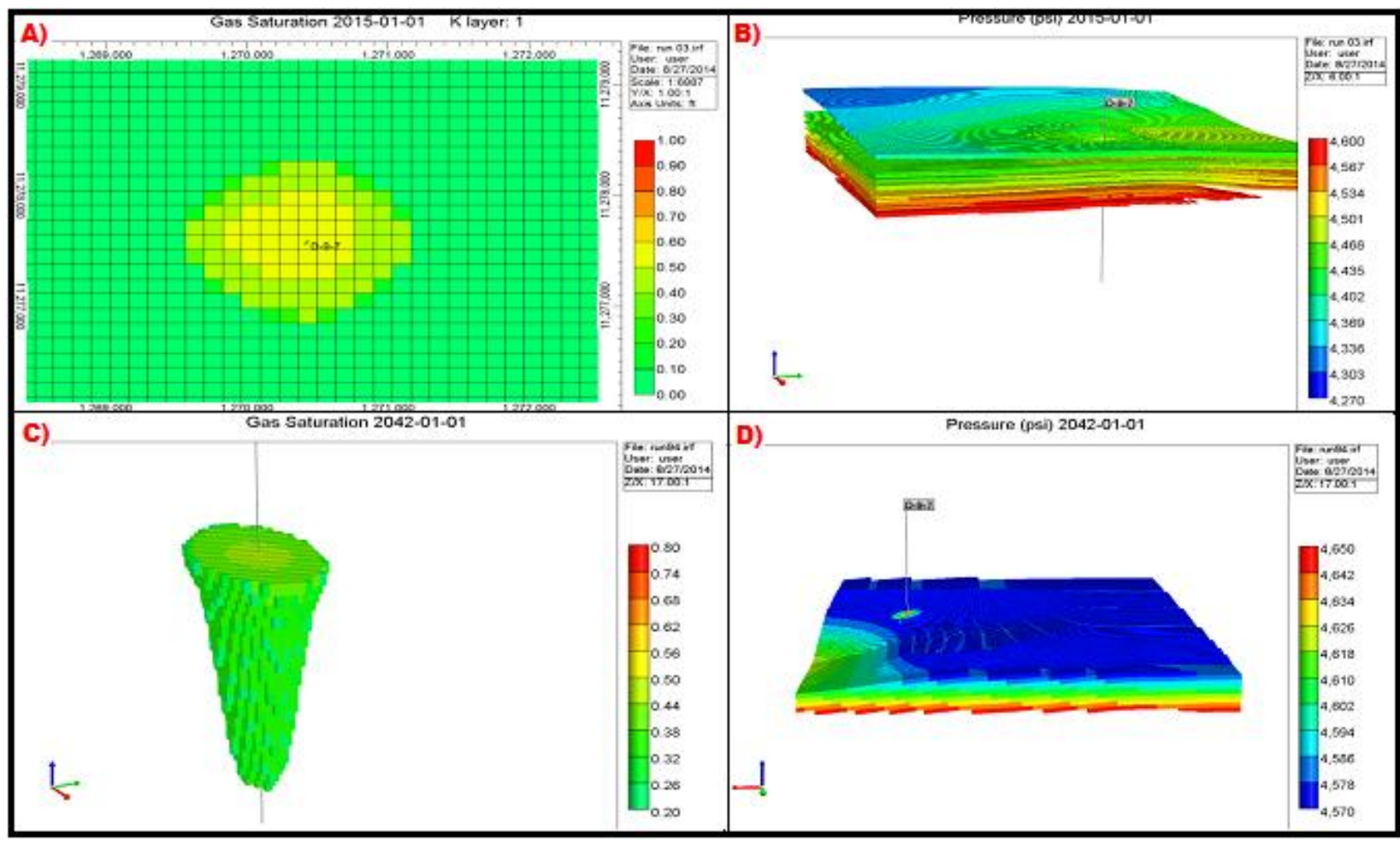

Figura 3. Distribución de la saturación de $\mathrm{CO} 2$ (A) y la distribución de presión (B) al final de los 3 años de inyección para el modelo ajustado históricamente. Distribución de la saturación de $\mathrm{CO} 2$ (C) y la distribución de presión (D) al final de los 30 años de inyección para el modelo sobredimensionado.

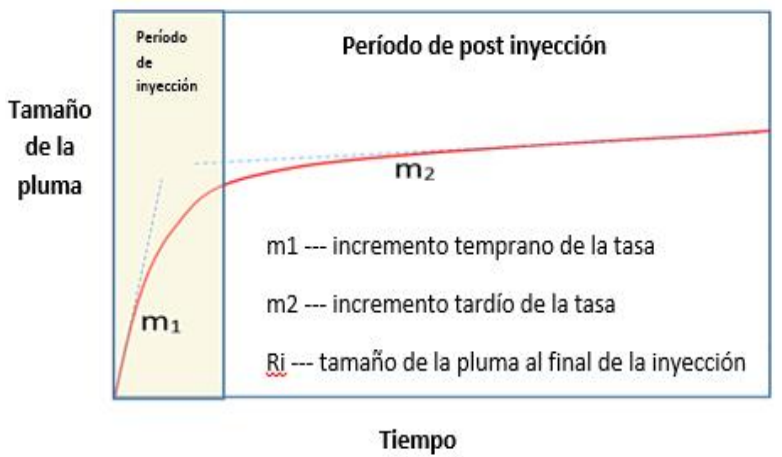

Figura 4. Tiempo de evolución de la pluma de saturación de $\mathrm{CO}_{2}$
3.1.2. Pluma de presión: Se seleccionaron varios umbrales de presión para estudiar el área de cobertura e influencia de la pluma. El tamaño de la pluma de presión refleja un comportamiento incremental al inicio de la inyección hasta alcanzar su punto más alto, que por lo general sucede en algún momento después de culminada la inyección. Después de eso, el tamaño de la pluma de presión decreció a una velocidad diferente dependiente de las condiciones de frontera aplicadas. En la figura $5 \mathrm{~b}$ se muestra el comportamiento de la pluma de presión: 

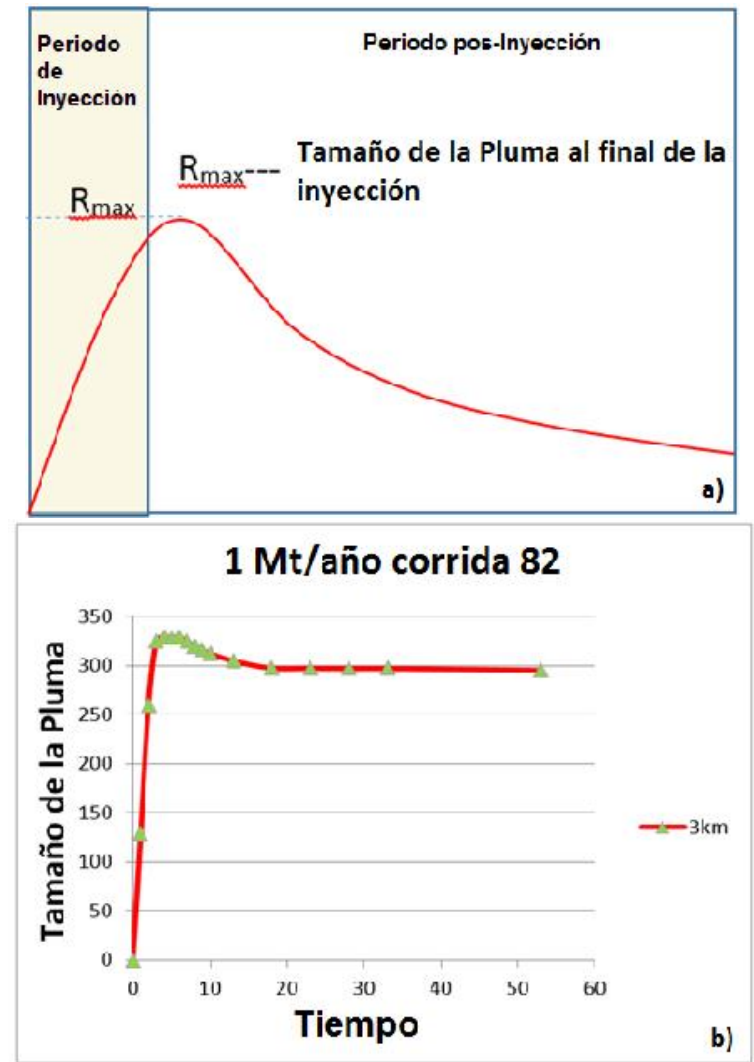

Figura 5. (a) Tiempo de evolución de la pluma de presión para un umbral específico en un sistema cerrado. . (b) Esquema general del comportamiento de la pluma de presión.

3.1.3. Presión a una ubicación específica en el reservorio: El tamaño de la pluma de presión fue analizada a diferentes distancias desde el punto de inyección: 1, 2 y $3 \mathrm{~km}$. El perfil de presión muestra un aumento rápido durante la inyección alcanzando su valor máximo al final del período de inyección. Posterior a esto la inyección se detiene y la presión comienza a decaer. Este comportamiento depende de la distancia del punto de inyección y de la heterogeneidad del yacimiento (Figura 6):

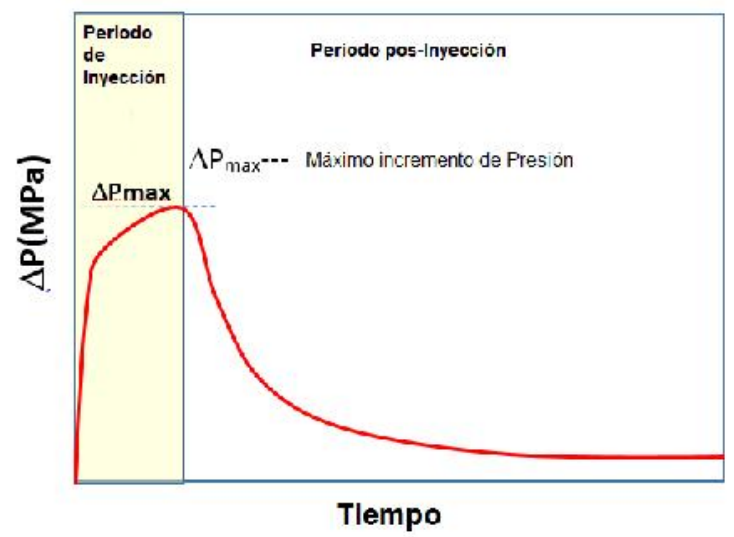

Figura 6. Tiempo de evolución de la presión en un punto determinado en el reservorio.

El análisis del comportamiento de la presión durante se llevó a cabo para los tres años de la inyección y cincuenta años de post-inyección, esto, con el objetivo de investigar la dinámica de los aumentos de presión a 1, 2 y 3 kilómetros de distancia desde el punto de inyección. Comparando todos los casos donde la tasa de inyección es cercana a 50,000 toneladas por año, vemos claramente que la presión aumenta rápidamente en diferentes lugares y disminuye repentinamente después de cerrar el pozo inyector. La estabilización de la presión se alcanzó 20 años después de la inyección aproximadamente (Figura 7):

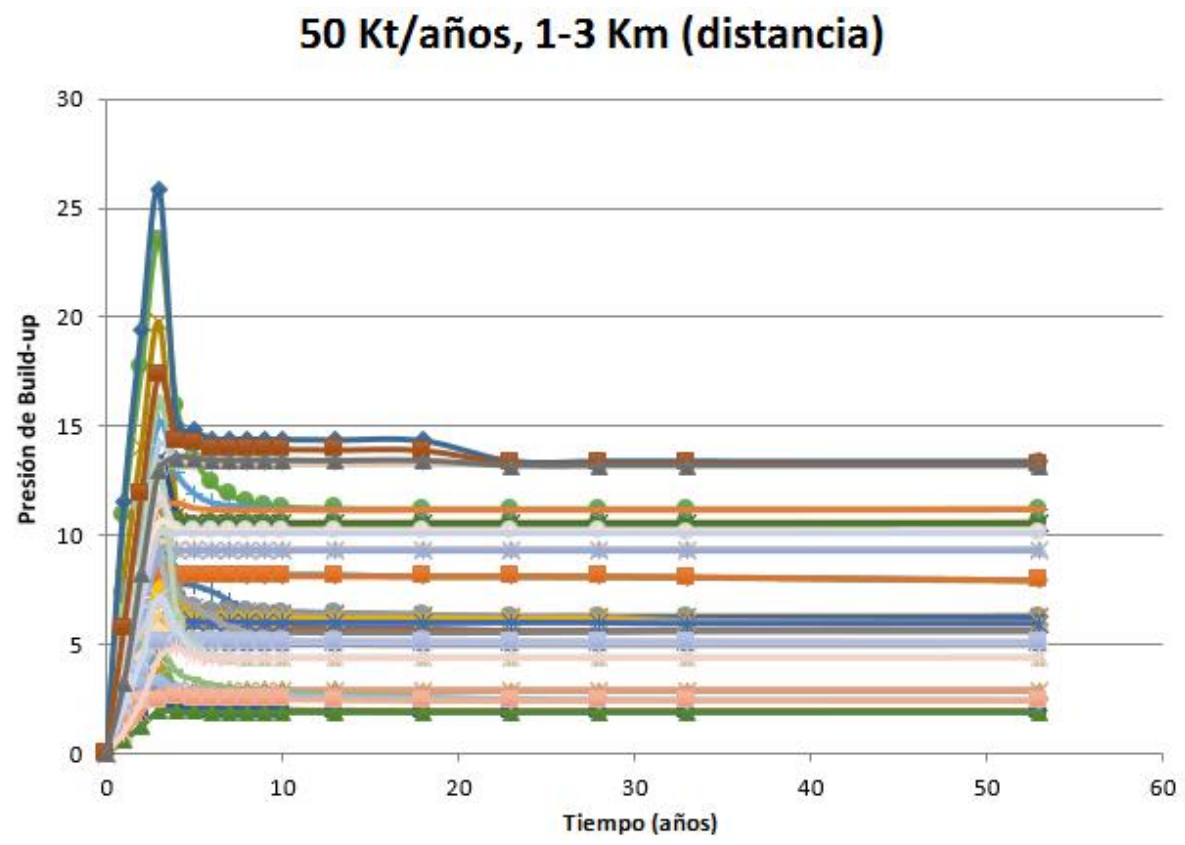

Figura 7. Incremento de presión vs tiempo a 1, 2 y 3 km de distancia desde el punto de inyección para un sistema cerrado. 


\subsection{Obtención y Análisis de resultados}

Se implementó un análisis de sensibilidad de factores para ambos modelos, con el fin de determinar los parámetros más importantes que afectan al tamaño de la pluma de saturación y de presión. Este análisis incluye la determinación de los parámetros pesados (de mayor importancia) ejecutando un diseño experimental de Plackett-Burman (PB), análisis del comportamiento no lineal de los parámetros más importantes y, finalmente, la generación de respuestas de superficie. Para llevar a cabo el diseño experimental se utilizó el software Minitab.

3.2.1 Análisis detallado del comportamiento del yacimiento: Para obtener un análisis convencional e intuitivo de estos resultados de simulación se grafica el tamaño de la pluma de presión y de saturación versus la tasa de inyección. En este análisis se esperaba que a medida que la tasa de inyección aumente, la pluma de presión también aumente; sin embargo, ya que los resultados de simulación involucran múltiples variables con correlaciones directas y cruzadas, la respuesta no siempre fue intuitiva. Ciertas simulaciones con una alta tasa de inyección presentaron tamaños de pluma más pequeños debido a un alto espesor de formación, y a valores de porosidad y de permeabilidad bajos; existen ciertas simulaciones con tamaños de pluma extensos, con una tasa baja de inyección, una alta permeabilidad y valores bajos de porosidad y espesor. Por lo tanto, se requirió la obtención de un número sin dimensiones que representara el impacto global de las diferentes variables y su correlación con la respuesta de simulación.

Se definió un número sin dimensiones " $\Psi$ " incluyendo los parámetros más importantes obtenidos a partir del diseño experimental de PB. La Figura 8 muestra la respuesta de simulación vs el número adimensional obtenido. Se observa una tendencia lineal clara cuando el tamaño de la pluma de saturación es graficado vs el número adimensional.

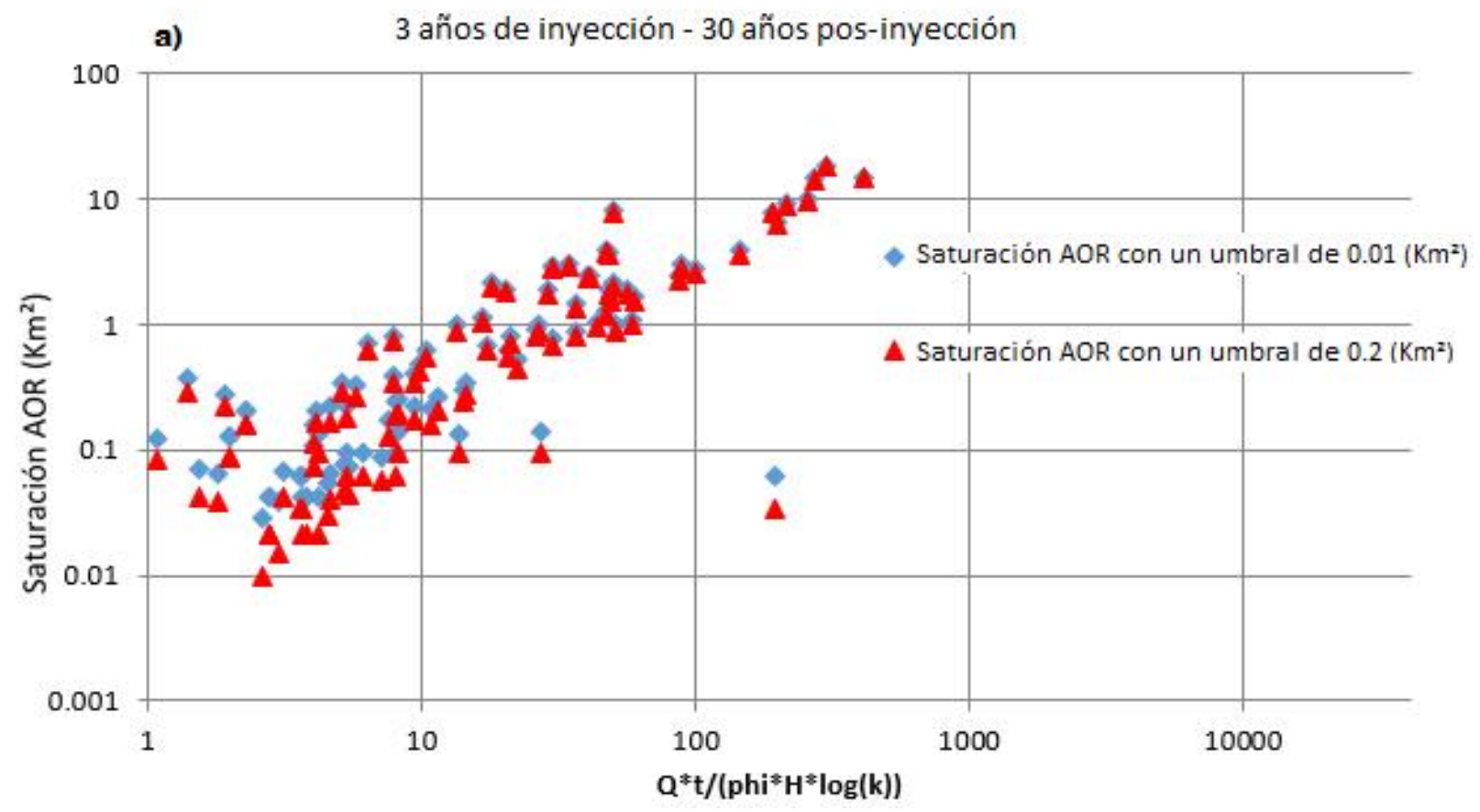

Figura 8. Tamaño de la pluma de saturación vs número adimensional utilizando el modelo sobredimensionado para un sistema cerrado.

La definición del número adimensional $\Psi$ es:

$$
\Psi=(\mathrm{q} \times \mathrm{t}) /(\varnothing \times \mathrm{h} \times \log ((\mathrm{k})) \quad \text { Ec. } 2
$$

El análisis de sensibilidad se realizó para ver los efectos de los diferentes parámetros en el número adimensional $\Psi$ como se muestra en la Figura 9.
3.2.2 Diseño Experimental de Plackett-Burman (PB): Un enfoque sistemático se aplicó para ambos modelos de yacimientos con el fin de encontrar los parámetros más importantes que afectan a la dinámica de la saturación de $\mathrm{CO}_{2}$ y al tamaño de la pluma de presión. 


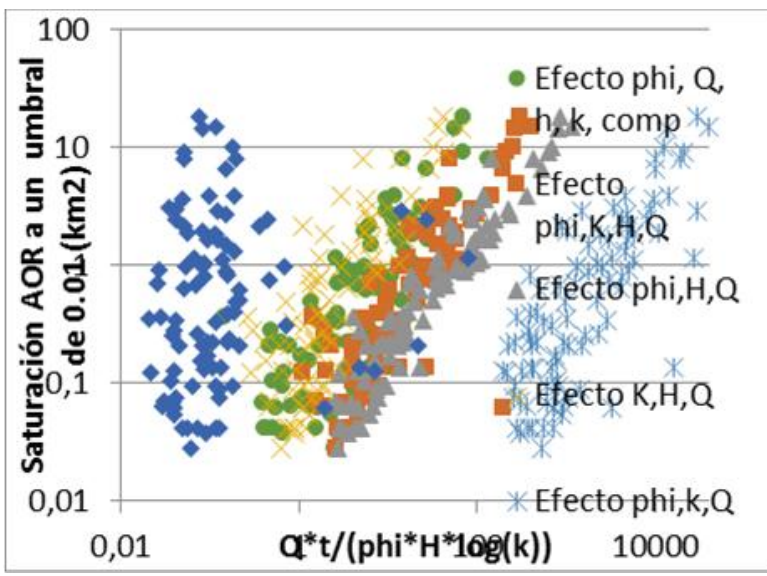

Figura 9 Relación lineal entre el número adimensional y las métricas de riesgo

El diseño de Plackett-Burman (PB) utilizado para este análisis, es un diseño de dos-niveles que requiere $(n+$ 1) simulaciones, en donde $n$ es el número de factores o variables. La tabla 1 muestra los 7 parámetros seleccionados para el análisis y su nivel de variación; sin embargo, con el objetivo de generar una escala de importancia de mayor resolución, en lugar de 8 simulaciones, se generó una matriz de diseño con 12 simulaciones para ambos modelos. Todas las columnas de la Tabla 2 son ortogonales entre sí. Las tablas 1 y 2 muestran la terminología de la matriz de diseño para los modelos de ajuste histórico y el modelo sobredimensionado, en las que el máximo valor de los factores es representado con (1), y el mínimo valor con $(-1)$.

Tabla 1. Parámetros usados en el diseño de PB

\begin{tabular}{|c|c|c|c|}
\hline Parámetro & $\mathbf{- 1}$ & $\mathbf{+ 1}$ & Unidad \\
\hline $\begin{array}{c}\text { A: } \\
\text { Permeabilidad }\end{array}$ & 10.19 & 977.224 & $\mathrm{Md}$ \\
\hline B: Kv/Kh & $\begin{array}{c}0.01065 \\
4\end{array}$ & $\begin{array}{c}0.99472 \\
8\end{array}$ & Adimen \\
\hline C: Porosidad & 0.05017 & 0.34789 & $\%$ \\
\hline D: Espesor & 50 & 200 & $\mathrm{M}$ \\
\hline $\begin{array}{c}\text { E: } \\
\text { Compresibilida } \\
\text { d }\end{array}$ & $\begin{array}{c}1.06 \mathrm{E}- \\
06\end{array}$ & $\begin{array}{c}1.01 \mathrm{E}- \\
05\end{array}$ & $1 / \mathrm{psi}$ \\
\hline $\begin{array}{c}\text { F: Salinidad } \\
\text { G: Tasa de } \\
\text { inyección }\end{array}$ & $\begin{array}{c}11.3709 \\
6\end{array}$ & $\begin{array}{c}228.121 \\
0\end{array}$ & $\mathrm{Ppm}$ \\
\hline
\end{tabular}

Tabla 2. Diseño de $P B$ para 7 variables, -1 = mínimo valor, +1 = máximo valor, 12 simulaciones .

\begin{tabular}{|c|c|c|c|c|c|c|c|}
\hline & Factor 1 & $\begin{array}{c}\text { Factor } \\
\mathbf{2}\end{array}$ & Factor 3 & Factor 4 & Factor 5 & Factor 6 & Factor 7 \\
\hline Corrida & $\begin{array}{c}\text { A: } \\
\text { Permeabilidad }\end{array}$ & $\begin{array}{c}\text { B: } \\
\mathbf{k v} / \mathbf{k h}\end{array}$ & $\begin{array}{c}\mathbf{C}: \\
\text { Porosidad }\end{array}$ & $\begin{array}{c}\mathbf{D}: \\
\text { Espesor }\end{array}$ & $\begin{array}{c}\text { E: } \\
\text { Compresibilidad }\end{array}$ & $\begin{array}{c}\text { F: } \\
\text { Salinidad }\end{array}$ & $\begin{array}{c}\text { G: Tasa de } \\
\text { Inyección }\end{array}$ \\
\hline & mo & $\mathbf{N A}$ & $\mathbf{\%}$ & $\mathbf{m}$ & $\mathbf{1 / p s i}$ & $\mathbf{p p m}$ & $\mathbf{k t} / \mathbf{y r}$ \\
\hline $\mathbf{1}$ & -1 & 1 & 1 & -1 & 1 & -1 & -1 \\
\hline $\mathbf{2}$ & -1 & -1 & 1 & 1 & 1 & -1 & 1 \\
\hline $\mathbf{3}$ & -1 & 1 & 1 & 1 & -1 & 1 & 1 \\
\hline $\mathbf{4}$ & 1 & -1 & -1 & -1 & 1 & 1 & 1 \\
\hline $\mathbf{5}$ & 1 & 1 & -1 & 1 & -1 & -1 & -1 \\
\hline $\mathbf{6}$ & -1 & -1 & -1 & -1 & -1 & -1 & -1 \\
\hline $\mathbf{7}$ & 1 & 1 & 1 & -1 & 1 & 1 & -1 \\
\hline $\mathbf{8}$ & 1 & -1 & 1 & -1 & -1 & -1 & 1 \\
\hline $\mathbf{9}$ & -1 & 1 & -1 & -1 & -1 & -1 & 1 \\
\hline $\mathbf{1 0}$ & 1 & 1 & -1 & 1 & 1 & 1 & -1 \\
\hline $\mathbf{1 1}$ & 1 & -1 & 1 & 1 & -1 & 1 & -1 \\
\hline $\mathbf{1 2}$ & -1 & -1 & -1 & 1 & 1 & & 1 \\
\hline
\end{tabular}

3.2.3 Diagrama de Pareto y Gráfico de Normalidad: El diagrama de Pareto muestra el tamaño relativo de los efectos y su contribución en las respuestas de simulación, es decir, la presión y la pluma de saturación. La variable tiene un impacto significativo en la respuesta de simulación, si cae por sobre una línea definida, basada en los intervalos de confianza; la variable no tiene un impacto significativo en los resultados de simulación si cae por debajo de esta línea. Las figuras 10-12 muestran que la permeabilidad del yacimiento y la tasa de inyección tuvieron una influencia significativa en el tamaño de la pluma de presión y de saturación para todos los umbrales y condiciones de frontera asignados.

Los gráficos de normalidad indican el efecto de polaridad de cada variable. En la figura 10 se observa que el efecto estándar de la permeabilidad del yacimiento se encuentra hacia la derecha de la línea normal, es decir, el efecto es positivo a la respuesta de simulación; en una formación de mayor permeabilidad, la pluma de saturación se expandirá más rápido. Estos gráficos además muestran que tanto la permeabilidad del yacimiento como la tasa de inyección y la 
compresibilidad de la roca, tienen correlación positiva con el tamaño de la pluma de saturación mientras que la porosidad tiene una correlación negativa.
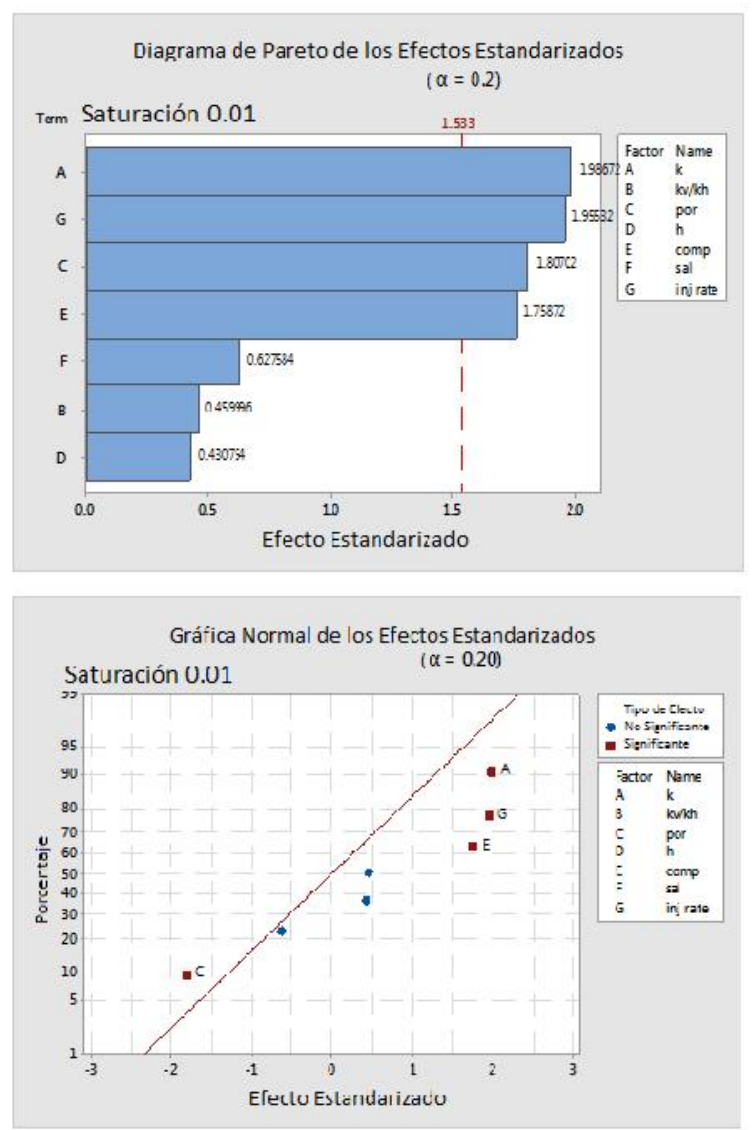

Figura 10 Diagramas de Pareto y gráficos de normalidad para el modelo sobredimensionado para un sistema cerrado.

El mismo análisis se llevó a cabo usando el tamaño de la pluma de presión como respuesta de simulación, con umbrales de presión de 1 y 5 bar.

Con el objetivo de aumentar significativamente la resolución de los resultados de simulación, se utilizó una técnica de pliegue en la matriz generada inicialmente por el diseño experimental de PB. Esta técnica consiste en un cambio de signos en todas las columnas de la matriz que se muestra en la tabla 2 [9]. La figura 12 presenta el diagrama de Pareto obtenido a partir de la técnica de pliegue y muestra concordancia con los análisis previos.

3.2.3.1. Efectos de condición de frontera: Para investigar el impacto de las condiciones de frontera sobre las respuestas de simulación se ejecutó una condición de frontera cerrada y una condición de frontera semi-abierta en el modelo de ajuste histórico. Se definió un sistema cerrado con ausencia de flujo a sus alrededores mediante el uso de una roca sello como barrera impermeable en el límite superior del yacimiento y una capa de baja permeabilidad en la frontera sur. Para el sistema semi-abierto, se aplicaron las mismas condiciones de impermeabilidad en las fronteras norte y sur, y una condición de flujo en las demás fronteras mediante el uso de un acuífero que rodea el sitio de almacenamiento de $\mathrm{CO}_{2}$.
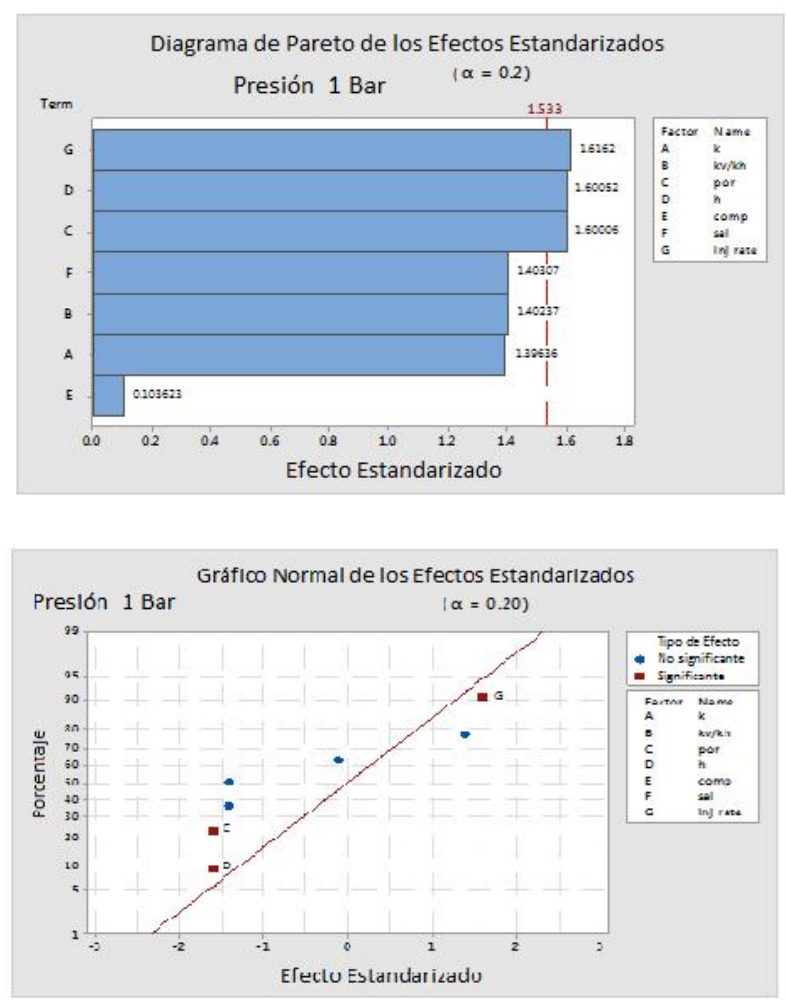

Figura 11 Diagramas de Pareto y gráficos de normalidad para el modelo sobredimensionado para un sistema cerrado a Presión de 1 bar

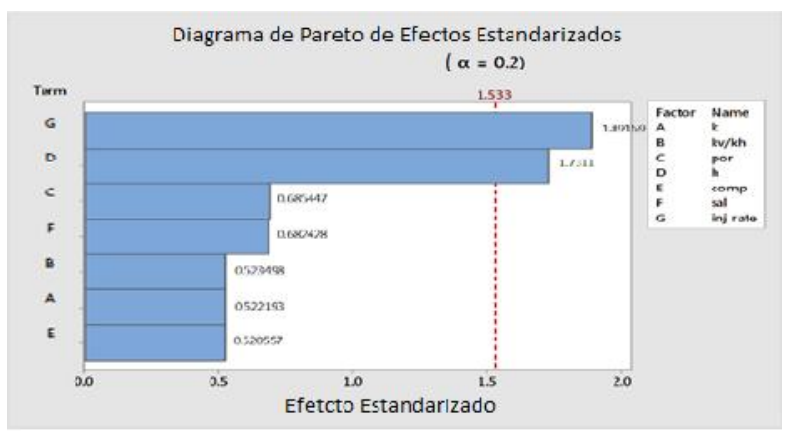

Figura 12 Diagrama de Pareto para el modelo sobredimensionado usando umbrales de presión de 5 bar.

La tabla 3 muestra el diseño de Plackett-Burman usado para comparar estas dos condiciones de frontera: 
Tabla 3. Rendimiento de las métricas de riesgo usando el modelo de ajuste histórico para analizar la condición de frontera

\begin{tabular}{|c|c|c|c|c|c|c|c|}
\hline \# Corridas & $\begin{array}{c}\text { Tiempo de } \\
\text { Inyeción } \\
\text { (Años) }\end{array}$ & $\begin{array}{c}\text { Tasa de } \\
\text { Inección } \\
\text { (Kt/año) }\end{array}$ & $\begin{array}{c}\text { Tiempo de } \\
\text { Pos } \\
\text { Inyección } \\
\text { (años) }\end{array}$ & $\begin{array}{c}\text { Compresibili } \\
\text { dad (1/psi) }\end{array}$ & $\begin{array}{c}\text { Tipo de } \\
\text { Frontera }\end{array}$ & Salinidad & kv/kh \\
\hline 1 & 3 & 10 & 30 & 0.0000101 & $\begin{array}{c}\text { Semi- } \\
\text { abierto }\end{array}$ & 11.37 & 0.994728 \\
\hline 2 & 3 & 25 & 30 & 0.0000101 & $\begin{array}{c}\text { Semi- } \\
\text { abierto }\end{array}$ & 11.37 & 0.994728 \\
\hline 3 & 3 & 10 & 30 & 0.0000101 & $\begin{array}{c}\text { Semi- } \\
\text { abierto }\end{array}$ & 228.121 & 0.010654 \\
\hline 4 & 3 & 10 & 30 & 0.0000106 & $\begin{array}{c}\text { Semi- } \\
\text { abierto }\end{array}$ & 228.121 & 0.994728 \\
\hline 5 & 3 & 25 & 30 & 0.0000101 & Cerrado & 228.121 & 0.010654 \\
\hline 6 & 3 & 25 & 30 & 0.0000106 & $\begin{array}{c}\text { Semi- } \\
\text { abierto }\end{array}$ & 228.121 & 0.010654 \\
\hline 7 & 3 & 10 & 30 & 0.0000101 & Cerrado & 11.37 & 0.010654 \\
\hline 8 & 3 & 10 & 30 & 0.0000106 & Cerrado & 11.37 & 0.010654 \\
\hline 9 & 3 & 10 & 30 & 0.0000106 & Cerrado & 228.121 & 0.994728 \\
\hline 10 & 3 & 25 & 30 & 0.0000101 & Cerrado & 228.121 & 0.994728 \\
\hline 11 & 3 & 25 & 30 & 0.0000106 & Cerrado & 11.37 & 0.994728 \\
\hline 12 & 3 & 25 & 30 & 0.0000106 & $\begin{array}{c}\text { Semi- } \\
\text { abierto }\end{array}$ & 11.37 & 0.010654 \\
\hline
\end{tabular}

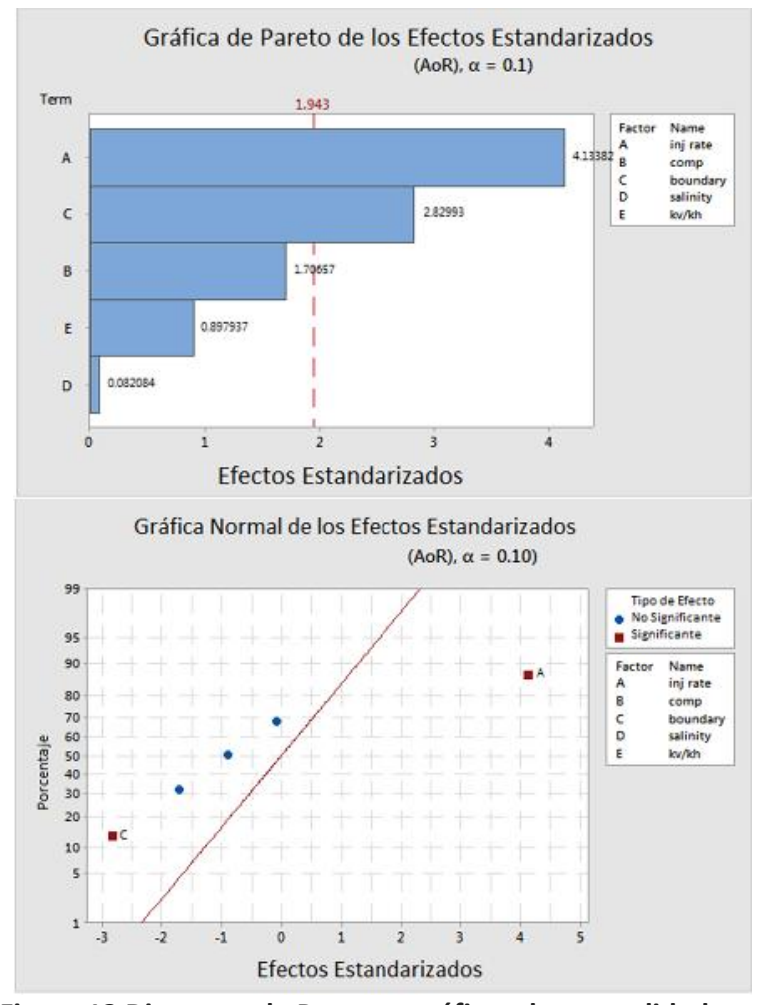

Figura 13 Diagrama de Pareto y gráficos de normalidad en el modelo de ajuste histórico para un sistema cerrado

Como se observa en la figura 13, el diagrama de Pareto y los gráficos de normalidad describen la importancia y la correlación de cada parámetro que afecta las respuestas de saturación y la pluma de presión. La tasa de inyección y la condición de frontera muestran un impacto significativo utilizando la pluma extensiva de presión como respuesta de simulación; por otra parte, la tasa de inyección muestra una correlación positiva con el tamaño de la pluma y, la condición de frontera una correlación negativa. La barrera impermeable causa efecto en la extensión de la pluma y retarda la disipación de la presión en el yacimiento.

3.2.3.2 Respuestas de superficie: Las respuestas de superficie son utilizadas para analizar las relaciones entre los parámetros significativos obtenidos de los gráficos de Pareto con las respuestas de simulación. ${ }^{[9]}$ En la figura 14 se muestran las respuestas de superficie que fueron generadas con el objetivo de encontrar la regresión entre la porosidad, la permeabilidad y el tamaño de la pluma de presión con diferentes umbrales de presión utilizando el modelo de ajuste histórico en un sistema cerrado.

A continuación, se presenta una evaluación cualitativa de las condiciones geológicas y operacionales de la investigación, en base a las simulaciones y al análisis de incertidumbre. La tabla 4 muestra los detalles de la evaluación cualitativa. 

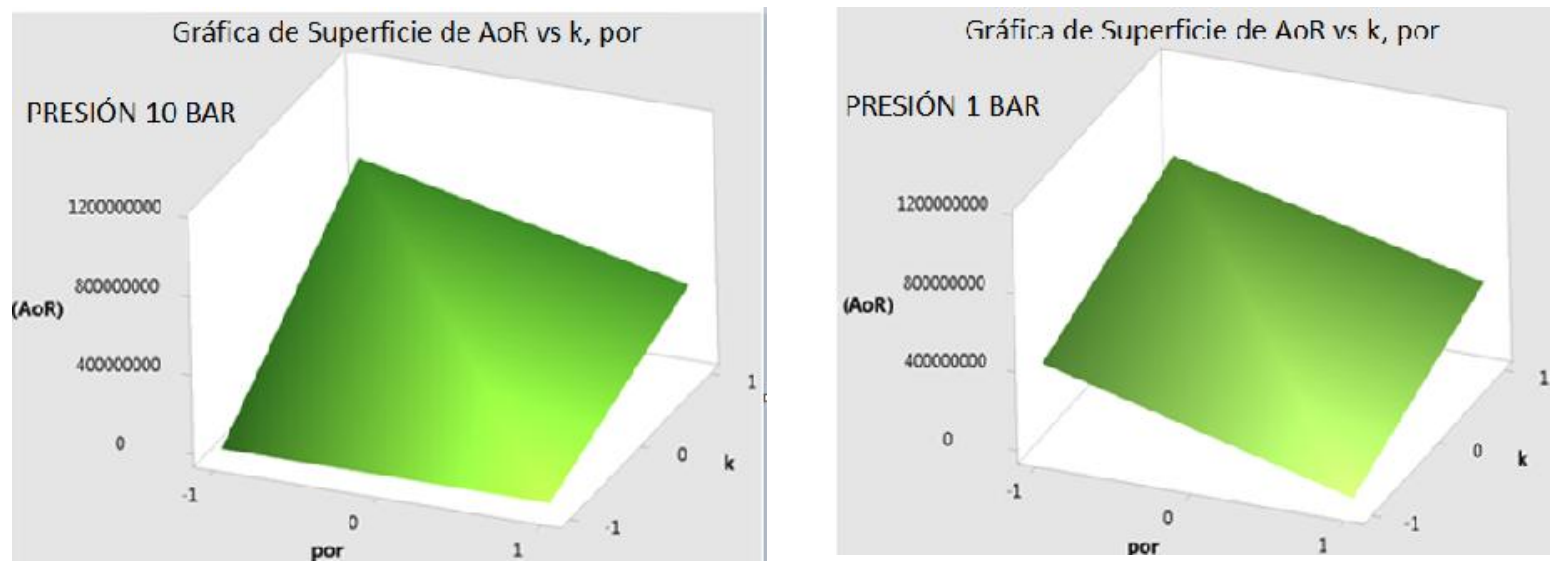

Figura 14 Gráficos de respuestas de superficie de simulación vs porosidad y permeabilidad para diferentes umbrales de presión y saturación del modelo sobredimensionado.

Tabla 4. Evaluación cualitativa de los parámetros

\begin{tabular}{|c|c|c|c|}
\hline Parámetro & $\begin{array}{l}\text { Impacto en } \\
\text { la Presión }\end{array}$ & $\begin{array}{l}\text { Impacto en la } \\
\text { Saturación }\end{array}$ & Observaciones \\
\hline Porosidad & Medio & Alto & $\begin{array}{c}\text { Para sistemas cerrados, el impacto de la porosidad } \\
\text { puede ser mayor, depende del umbral de presión y de } \\
\text { la tasa de inyección }\end{array}$ \\
\hline Permeabilidad (k) & Alto & Alto & $\begin{array}{l}\text { Para sistemas cerrados, el impacto de la } \\
\text { permeabilidad puede ser menor, depende del umbral } \\
\text { de presión y de la tasa de inyección }\end{array}$ \\
\hline Compresibilidad & Bajo-Medio & Bajo & $\begin{array}{c}\text { La comprensibilidad tendrá un mayor impacto en la } \\
\text { presión para un sistema cerrado donde el volumen de } \\
\text { poro esté dentro de un orden de magnitud de } \\
\text { volumen de inyección }\end{array}$ \\
\hline Espesor & Bajo-Medio & Bajo-Medio & \\
\hline $\mathrm{Kh} / \mathbf{k v}$ & Bajo & Bajo-Medio & \\
\hline Salinidad & Bajo & Bajo & \\
\hline $\begin{array}{l}\text { Permeabilidad } \\
\text { Roca sello }\end{array}$ & Bajo-Medio & Bajo & $\begin{array}{c}\text { La permeabilidad de la roca sello tiene más impacto } \\
\text { cuando la masa inyectada no es demasiado grande y } \\
\text { la permeabilidad de la roca sello es baja }\end{array}$ \\
\hline $\begin{array}{l}\text { Condiciones de } \\
\text { Frontera }\end{array}$ & Medio-Alto & Bajo & $\begin{array}{l}\text { Las condiciones de fronteras son importantes para } \\
\text { mayores volúmenes de inyección o reservorios más } \\
\text { pequeños. }\end{array}$ \\
\hline
\end{tabular}

\section{Conclusiones}

El almacenamiento de $\mathrm{CO}_{2}$ en acuíferos salinos profundos requiere una comprensión avanzada de la roca del yacimiento, de las propiedades de los fluidos, y de las interacciones de diferentes condiciones geológicas y operacionales en la dinámica de los fluidos durante y después de la inyección de dióxido de carbono antropogénico $\left(\mathrm{CO}_{2}\right)$ Esto se resume en dos preguntas fundamentales:

El perfil de la pluma de $\mathrm{CO}_{2}$ aumentó durante el período de inyección y su tasa de crecimiento se estabilizó lentamente después de la inyección. La tasa de crecimiento después de la inyección depende de múltiples variables geológicas, operacionales y de sus correlaciones.

El perfil de la pluma de presión mostró un incremento repentino durante la inyección hasta que alcanzó su valor máximo antes de que la pluma comience a disminuir después de la inyección.

El perfil de la pluma de presión a distintos puntos cercanos al pozo de inyección, mostró un incremento rápido durante la inyección y una disminución rápida después de la inyección. 
La degradación de la pluma después de detener la inyección dura unos cuantos años, dependiendo de la cantidad de $\mathrm{CO}_{2}$ inyectado, de la porosidad y permeabilidad de la roca y de la condición de frontera.

La tasa de inyección, la permeabilidad del yacimiento y la condición de frontera mostraron un mayor impacto en el tamaño de la pluma de saturación y de presión.

\section{Nomenclatura}

$\Psi=$ Definición del número adimensional

$\emptyset=$ porosidad

$\mathrm{h}=$ espesor

$\mathrm{k}=$ permeabilidad

$\mathrm{kt} / \mathrm{año}=$ kilotoneladas por año

$\mathrm{Md}=$ milidarcy

$\mathrm{M}=$ metros

$\mathrm{PB}=$ Plackett-Burman

Ppm = partículas por millón

$\mathrm{q}=$ tasa de inyección

$\mathrm{t}=$ tiempo

\section{Referencias}

[1] Dennis YC, Leung, GC, Mercedes Maroto Valer M. An overview of current status of carbon di oxide capture and storage technologies. Renewable and Sustainable Energy Reviews. 2014; 39:426-443.

[2] Bachu, S., Bonijoly, D., Bradshaw, J., Burruss, R., Holloway, S., Christensen, N.P., Mathiassen, O.M., 2007. CO2 storage capacity estimation: methodology and gaps.
[3] Gibson-Poole, C., Edwards, S., Langford, R., Vakarelov, B., 2006. Review of Geological Storage Opportunities for Carbon Capture and Storage (CCS). Australian School of Petroleum, The University of Adelaide, Adelaide, South Australia, AU.

[4] M. Bentham and Mg. Kirby. $\mathrm{CO}_{2}$ Storage Saline Aquifers. Oil \& Gas Science and Technology Rev. IFP, 603 (2005) 559-567

[5] McKay, M.D., Beckman, R.J., Conover, W.J., 1979. A comparison of three methods for selecting values of input variables in the analysis of output from a computer code. Technometrics 21, 239-245.

[6] Koperna G.J, Kuuskraa V.A.,Reistenberg D.E, Rhudy R., Trautz R., Hill G.,Esposito R.A," The SECARB Anthropogenic Test: The First US Integrated Capture, Transportation, and Storage Test" Carbon Management Technology Conference, 7-9 February 2012, Orlando, Florida, USA

[7] Talabani, S., Boyd, D., Wazeer, F. El, \& Arfi, S. Al. (2000). Validity of Archie Equation in Carbonate Rocks. In Abu Dhabi International Petroleum Conference and Exhibition (pp. 643650).

[8] Alireza Haghighat, 2013 - Pressure History Matching for $\mathrm{CO}_{2}$ Storage in Saline Aquifers: Case Study for Citronelle Dome CARBON MANAGEMENT CONFERENCE

TECHNOLOGY

[9] Box, G. E. P., Hunter, W. G. and Hunter, J. S. (2005). Statistics for Experimenters: Design, Innovation, and Discovery. Wiley, New York. 